\title{
Stochastic Dynamics of Extended Objects in Driven Systems II: Current Quantization in the Low-Temperature Limit
}

\author{
Michael J. Catanzaro ${ }^{\mathrm{a}}$, Vladimir Y. Chernyak ${ }^{\mathrm{a}, \mathrm{b}}$, John R. Klein ${ }^{\mathrm{a}}$ \\ ${ }^{a}$ Department of Mathematics, Wayne State University, 656 W. Kirby, Detroit, MI 48202 \\ ${ }^{b}$ Department of Chemistry, Wayne State University, 5101 Cass Ave, Detroit, MI 48202
}

\begin{abstract}
Driven Langevin processes have appeared in a variety of fields due to the relevance of natural phenomena having both deterministic and stochastic effects. The stochastic currents and fluxes in these systems provide a convenient set of observables to describe their non-equilibrium steady states. Here we consider stochastic motion of a $(k-1)$-dimensional object, which sweeps out a $k$-dimensional trajectory, and gives rise to a higher $k$-dimensional current. By employing the low-temperature (low-noise) limit, we reduce the problem to a discrete Markov chain model on a CW complex, a topological construction which generalizes the notion of a graph. This reduction allows the mean fluxes and currents of the process to be expressed in terms of solutions to the discrete Supersymmetric Fokker-Planck (SFP) equation. Taking the adiabatic limit, we show that generic driving leads to rational quantization of the generated higher dimensional current. The latter is achieved by implementing the recently developed tools, coined the higher-dimensional Kirchhoff tree and co-tree theorems. This extends the study of motion of extended objects in the continuous setting performed in the prequel [6] to this manuscript.
\end{abstract}

\section{Introduction}

Non-equilibrium thermodynamics is an area of statistical mechanics dealing with driven, and often time-varying, systems evolving via chemical reactions and exchange of energy $[16,20,18,17,36]$. These systems can be accurately described by a Langevin equation when the typical time scale of the system is slow compared to the relaxation time of the coupled bath [23, 15, 33]. Because of the generality of this setup, Langevin dynamics have found applications in a prodigious array of fields including chemistry $[23,17]$, biology $[2,3]$, finance $[5,26,21]$, and certain engineering applications $[14,9,27]$. It is the ubiquitous nature of the Langevin framework that allows such a pervasive grasp across the physical sciences.

Within the framework of non-equilibrium thermodynamics, the notion of stochastic current, or flux, has recently gathered interest as a compelling observable which gives insight on the underlying system [35, 32, 1, 31, 10]. Together with the probability density, stochastic currents provide an adequate set of observables for describing the long-time behavior of probability distributions in this setting. A stochastic flux can be measured by counting how many times the system spans a cycle, or equivalently, by counting intersections of a stochastic trajectory with a cross section. This notion of current has been developed completely in the discrete setting on a mathematical graph, in which case the components of the stochastic current are simply given by counting how many times a stochastic trajectory crosses the edge. In the discrete case of graphs, this Langevin process can be reduced to a continuous-time Markov chain. The goal of this manuscript is to extend the concept of stochastic trajectory to higher dimensions, as well as the underlying graph on which the trajectory lies.

This manuscript constitutes the sequel to our work on higher-dimensional Langevin processes on continuous state spaces of arbitrary dimension. In the prequel [6], we explained why the proper way of defining a Langevin process in this setting is in terms of stochastic vector fields on the underlying state space $X$. Not only does this generalize the usual definition in a natural way, it allows us to take full advantage of the dynamical symmetry which is necessary for the reduction to the discrete model discussed here. After giving several examples of higher dimensional currents, we defined the generalized Supersymmetric Fokker-Planck (SFP) operator and equation, which governs the evolution of higher-dimensional objects on $X$. Using this equation, we were able to write an expression for the average current generated under a Langevin process. This expression was then re-cast, in the adiabatic limit, with the solution to the higher dimensional Kirchhoff network problem and the higher-dimensional Boltzmann distribution. While all of this was performed in the continuous case, the low-temperature (or low-noise) limit connects the two manuscripts, as the continuous dynamics tend to a discrete structure naturally embedded in $X$. In this way, one interested in these two limits can forget the complicated continuous picture and focus on the simpler, more computational discrete setting.

The manuscript is organized as follows. Section 2 serves 
as a summary of the main ideas and specialized tools used throughout our calculations. In section 3, we describe how the low-noise limit allows for a reduction of the stochastic process to a Markov chain model defined on the MorseSmale complex, defined there. Section 4 contains a formal expression for the average current generated by a Langevin process. The higher-dimensional Kirchhoff network theorem is defined and solved in 4.2, whereas the dual higher Boltzmann distribution is given in 4.3. These two results are combined in subsection 4.4 to give a much more computable form of the generated current. This version of the current is used in section 5 to prove the main result of the manuscript: for generic driving in the low-temperature, adiabatic limit, the generated current is rationally quantized.

\section{Technical Introduction}

In this section, we present the main results and concepts of the manuscript. Our calculations are technical, and since we do not assume any topological background, this section serves as an instructive and brief bridge to our results.

The section is organized as follows. Subsection 2.1 contains a simple and self-contained presentation of the structures needed for a description of higher-dimensional discrete stochastic models: CW complex, Morse complex, and CW complex homology. We also provide an initial insight into how these discrete models, associated with CW complexes, arise naturally from continuous models in the long-time, low-noise limit. In subsection 2.2 we present the main results of the manuscript on fluxes in higherdimensional discrete models: higher-dimensional versions of the tree and co-tree Kirchhoff theorems, explicit expressions for fluxes, and quantization of higher-dimensional fluxes in the low-temperature adiabatic limit for periodic potential driving.

\subsection{CW complexes, Morse Complex, and Slow Low- Temperature Limit for Stochastic Dynamics}

As briefly discussed in section 1 , if one is interested in the low-temperature (low noise) limit on long time scales, stochastic dynamics on continuous spaces can be reduced to its discrete counterpart. In the simplest case of a potential force (often referred to as a gradient flow), the discrete setting starts with a graph whose vertices are represented by the local minima of the potential $V(x)$, whereas the edges correspond to saddle points, or more rigorously to critical points of the potential (defined by the conditions $d V=0)$ with exactly one unstable mode. Equivalently, these are critical points with Morse index (which is the number of unstable modes) equal to 1 . On the initial time scales of low-noise stochastic dynamics, a particle approaches a local minimum, completely determined by the deterministic component $u(x)$ of the velocity field in the Langevin equation (Eq. (1) of [6]). After that, the particle will spend most of the time in a small neighborhood of the local minimum, participating in rare transitions. These occur when a highly improbable configuration of the noise field takes the particle to a saddle point, and actually a little bit past it, so that it further drops to a different local minimum with no additional noise assistance needed. The rate $k_{a \alpha} \sim e^{-\kappa^{-1}\left(V\left(x_{\alpha}\right)-V\left(x_{a}\right)\right)}$ of a transition over the edge $\alpha$ that starts at vertex $a$ can be calculated analytically, including the pre-exponential factor, using the theory of rare events [24]. Therefore, long-time stochastic dynamics are adequately described by a continuous-time Markov chain process on the graph introduced above. It can be described by a Master Equation (ME), with the spectrum of the Master Operator (MO) representing the soft modes of the spectrum of the Fokker-Planck (FP) operator, the latter describing Langevin dynamics in $X$. Note that the graph is naturally embedded into $X$, with its edges representing the most probable transition trajectories (also known as optimal fluctuations or instantons) that dominate the probabilities of the transition events. It is intuitively natural that in the case when one follows the low-noise evolution of higher-dimensional objects, the graph should be replaced by a higher-dimensional object. Such an object is known as a $C W$ complex, and plays an important role in differential and algebraic topology; our application deals with a special example of a CW complex, namely the Morse complex of a gradient flow.

In what follows we describe how a CW complex generalizes a notion of a graph to higher dimensions. One can think about a graph as built in the following way. We start with a set $K_{0}$ of the graph vertices, or 0-dimensional cells; they form the 0 -skeleton $K^{(0)}$. We further consider the set $K_{1}$ of edges, or 1-dimensional cells, with each edge being represented by a segment. Edges are attached to the 0 -skeleton along their boundaries, represented by pairs of points; this forms the 1-skeleton $K^{(1)}$, which is the same as a graph. Stated differently, if we stopped at this step, then we would be studying graphs. However, we can go further and consider a set $K_{2}$ of 2-cells (we omit "dimensional") represented by 2 -disks $D^{2}$ and attach them to the 1-skeleton along their boundaries, represented by circles $S^{1}$, by means of maps $S^{1} \rightarrow K^{(1)}$, referred to as attaching maps. If we continue the procedure attaching $i$-discs to the $(i-1)$-skeleton along their boundaries, represented by $(i-1)$-spheres $S^{i-1}$ and eventually stop at the $m$-skeleton, as a result we build a space called an $m$-dimensional $\mathrm{CW}$ complex. If a space $X$ is represented by a CW complex $K$, we call the latter a $C W$-decomposition of $X$. We emphasize that within the described terminology, a graph is nothing more than a 1-dimensional CW complex. Also note that each manifold of dimension $D$ can be triangulated, i.e., partitioned into a set of triangles for $D=2$, tetrahedra for $D=3$, etc. A triangulation is the simplest example of a CW-decomposition, with its structure being transparent and obvious. For example, a 3-dimensional manifold has its 3-skeleton as the manifold itself, the 21- and 0-skeletons are represented by the unions of the 
tetrahedra faces, edges, and vertices, respectively.

The concept of higher-dimensional current on a $\mathrm{CW}$ complex, which generalizes the notion of current on a graph, is most naturally formulated in terms of the chain complex of a CW complex. An $n$-chain is a linear superposition of $n$-cells with integer or real coefficients; the spaces of $n$-chains are denoted $C_{n}(K)$ or $C_{n}(K ; \mathbb{R})$ respectively. The boundary operator $\partial_{n}: C_{n} \rightarrow C_{n-1}$ maps an $n$-cell to a linear superposition of $(n-1)$-cells, completely determined by the attaching maps. The integer coefficients characterize how many times (accounting for orientations) the boundary of the $n$-cell covers the relevant $(n-1)$-cell. In our applications, we deal with regular CW complexes, i.e., the ones for which the coefficients in the aforementioned superpositions are \pm 1 or 0 . Note that a triangulation is a regular $\mathrm{CW}$ complex. The inclusion $B_{n} \subset Z_{n} \subset C_{n}$, with $B_{n}=\operatorname{im}\left(\partial_{n+1}\right)$ and $Z_{n}=\operatorname{ker}\left(\partial_{n}\right)$ referred to as $n$-boundaries and $n$-cycles, reiterates the intuitive and natural fact that the boundary of something is a cycle, i.e., $\partial \circ \partial x=0$ for any $x$ and in any dimension. The aforementioned inclusion allows CW-homology to be defined by setting $H_{n}(K)=Z_{n}(K) / B_{n}(K)$, or over real coefficients $H_{n}(K ; \mathbb{R})=Z_{n}(K ; \mathbb{R}) / B_{n}(K ; \mathbb{R})$. The importance of CW-homology is that it gives a computationally accessible handle on the homology of the underlying space, i.e., if $K$ is a $\mathrm{CW}$-decomposition of $X$, then $H_{i}(X) \cong H_{i}(K)$, and $H_{i}(X ; \mathbb{R}) \cong H_{i}(K ; \mathbb{R})$. With integer coefficients, this is valid for the singular homology of $X$ also. With real coefficients, it works also for a broader class of homology, e.g., bordism homology considered in $[6]$.

The Morse CW complex (decomposition) is a very natural construction that characterizes a gradient flow: the set $K_{n}$ of $n$-cells is represented by critical points of Morse index $n$. The gradient flow generates a $n$-cell out of a small neighborhood of the critical point, often referred to as the unstable manifold, naturally embedded into $X$ and attached to the $(n-1)$-skeleton; the latter is determined by the long-time behavior of the flow. If we are looking at the low-noise, stochastic motion of higher-dimensional cycles in the long-time limit, it can be reduced to a discrete stochastic model on the Morse CW complex, similar to how it is done in the simpler case of motion of points that is reduced to a Markov chain model on a graph (the latter being actually represented by the 1-skeleton of the Morse CW complex). The details of this reduction are presented in section 3 , here we provide a simple intuitive picture for the $n=2$ case, i.e., 1-dimensional cycles create 2-dimensional trajectories.

The short-time relaxation of an initial 1-cycle $\gamma: S^{1} \rightarrow$ $X$ forces it to approach the 1-skeleton. ${ }^{1}$ This defines a 1-chain $\bar{\gamma} \in C_{1}(K)$ of the Morse complex by counting how many times (with the proper \pm 1 sign weights, accounting for orientations) the relaxed cycle goes along the 1-cells

\footnotetext{
${ }^{1}$ In chemical physics this effect forms a basis for a number of algorithms for finding transition states in molecules.
}

(edges) of the 1-skeleton, represented by a graph. Longtime dynamics are represented by rare transitions when a fragment of the 1-cycle associated with some given edge goes over one of 2-cells it is attached to, replacing the initial fragment with the set of fragments that go over the rest of the edges the 2-cell is attached to. Such an effect is referred to as an elementary transition, because it generalizes the notion of a jump over an edge in a standard continuoustime Markov chain process. A sequence of elementary transitions naturally creates a 2 -chain $\bar{\eta}$ that characterizes a discrete counterpart of a stochastic trajectory. Averaging over stochastic trajectories and dividing by the total time of the process we obtain a map $Z_{1}(K) \rightarrow C_{2}(K ; \mathbb{R})$. If in the $t \rightarrow \infty$ limit, we have $t^{-1} \partial_{2} \bar{\eta} \rightarrow 0$, we refer to the situation as "the stochastic trajectories are statistically closed". This condition implies $t^{-1} \bar{\eta}$ is a cycle, and thus gives rise to a homology class $\left[t^{-1} \bar{\eta}\right]$. In this way, we obtain a linear map $H_{1}(K) \rightarrow H_{2}(K ; \mathbb{R})$, or in the general case a linear map

$$
\omega: H_{n-1}(K) \rightarrow H_{n}(K ; \mathbb{R})
$$

referred to as the flux map. Replacing the CW-homology of the Morse complex with the homology of the underlying space $X$, we obtain a flux map of the form considered in [6] for a continuous setting of stationary or periodic driving. We use the same notation for the both, since the flux map of Eq. (1) should reproduce its more general counterpart in the cases when the discrete stochastic dynamics on a CW complex adequately approximates the continuous stochastic dynamics on the underlying space.

Our formula for the average current density is given explicitly in terms of subcomplexes of $K$. A subcomplex $L$ of $K$ is a CW complex in its own right, together with an inclusion $L \subset K$ that preserves the relevant structure, i.e., attaching maps. A spanning tree (of dimension $d)$ is a subcomplex $T$ of $K$ so that $H_{d}(T)=0$ and $H_{i}(T ; \mathbb{R})=H_{i}(K ; \mathbb{R})$ for all $i<d$. This should be thought of as a crude approximation of $K$, since we have removed all $d$-dimensional 'loops', but nothing more. Every spanning tree acquires a weight as well, which arises in the formula for current density and quantization. The weight of a spanning tree $T$ is

$$
w_{T}=\theta_{T}^{2} \prod_{\alpha \in T_{d}} e^{-\kappa^{-1} W_{\alpha}},
$$

where the product is taken over all $d$-cells in $T$ and $\theta_{T}$ is the order of the torsion subgroup of $H_{d-1}(T)$. The number $\theta_{T}$ is a measure of the difference between $H_{d-1}(T)$ and $H_{d-1}(T ; \mathbb{R})$ and, so far as we can determine, has no physical meaning. In the analogy with resistive networks, each factor in this formula can be interpreted as the resistance of an edge, and therefore their product is the resistance of the network determined by $T$.

A spanning co-tree (of dimension $(d-1)$ ) is a subcomplex $L$ of $K$ for which $H_{i}(L ; \mathbb{R})=H_{i}(K ; \mathbb{R})$ for all $i<d$. These should be thought of as even cruder approximations of $K$ than spanning trees, since they can be given by 
brute truncation. In the case when $K$ is one-dimensional, and hence just a graph, spanning trees coincide with their classical counterparts, and spanning co-trees are just single vertices. Spanning co-trees also have a weight given by

$$
\tau_{L}=c_{L}^{2} \prod_{b \in L_{d-1}} e^{-\kappa^{-1} E_{b}},
$$

where the product is taken over all $(d-1)$-cells of the cotree, and $c_{L}$ is again given in terms of torsion subgroups of $H_{d-1}(X)$ and $H_{d-1}(L)$. The precise definition of $c_{L}$ is given in [7]; this definition sheds little insight physically and we omit it for clarity. The weights for both trees and co-trees fall out of constructing pseudo-inverses, and so one should think of them as the necessary factors for the current formula to be valid.

\subsection{The Main Results: Higher-Dimensional Kirchhoff Theorems and Quantization of Stochastic Currents}

The Kirchhoff network problem for graphs asks, given voltages in a resistive network, find the unique current flowing through the network. The first mathematically rigorous treatment of this problem was given by Hermann Weyl in 1923 [37], and an algebraic topological proof appeared shortly thereafter [25]. We aim to generalize this problem and its solution to arbitrary CW complexes.

We are passing from one description of dynamics (a smooth, cohomological one in terms of differential forms) to its dual (a discrete, homological picture in terms of chain complexes). The main object of study will now be the chain complex $C_{*}(K)$, instead of the Grassmann algebra, and so the operator $\mathcal{Q}$ is replaced with the differential $\partial$ on $C_{*}(K)$. The reduced dynamics on $K$ requires us to only know the values of the potential $V$ at the finite set of critical points; set $E_{i}:=V(i)$ and $W_{\alpha}:=V(\alpha)$ for each critical point of index $(n-1)$ and $n$, respectively. Finally, a driving protocol keeps track of this data; it assigns to each index $(n-1)$-critical point $i$, the real number $E_{i}$, and to each index $n$-critical point $\alpha, W_{\alpha}$. We are concerned with the non-stationary case, so the driving protocol will be a function of time.

The first quantities to translate to the discrete setting are the SFP operator and the current density. By constructing an adjoint $\partial^{\dagger}$ to the boundary operator $\partial$ in the obvious fashion, we obtain the discrete counterpart to the continuous SFP equation (Eq. (25) of in [6])

$$
\frac{d \rho}{d \tau}=-\partial J, \quad J=e^{-\kappa^{-1} \hat{W}(t)} \partial^{\dagger} e^{\kappa^{-1} \hat{E}(t)} \rho .
$$

We use $\rho$ for the distribution in the discrete, CW setting to distinguish it from the continuous distribution $\varrho$.

The main result of this paper concerns an equivalent formula for the current density $J$ which is computable, unlike the purely formal definition given above. The form combines the solution to Kirchhoff's higher network problem and the Boltzmann distribution in higher dimensions. Kirchhoff's problem can be solved using pseudo-inverses, even in the discrete case, with an operator analogous to $A_{V(\tau), \kappa}$ of [6]. Here, the operator $A_{\hat{W}(\tau), \kappa}$ acts on $(d-1)$ boundaries to produce $d$-chains. It is uniquely determined by the following two conditions:

$$
\partial A_{\hat{W}(t), \kappa} y=y, \quad\left(A_{\hat{W}(t), \kappa} y, z\right)_{\hat{W}(t), \kappa}=0,
$$

for any $y \in B_{d-1}(K ; \mathbb{R})$ and $z \in Z_{d}(K ; \mathbb{R})$. The modified inner product is defined to be

$$
(a, b)_{\hat{W}(t), \kappa}=\left(e^{\kappa^{-1} \hat{W}(t)} a, b\right),
$$

for any $a$ and $b$ in $C_{d-1}(K ; \mathbb{R})$. These two equations are the discrete analogues of Eqs. (34) and (33) from [6], respectively.

The Boltzmann distribution $\rho_{B}([\gamma] ; \hat{E}(\tau), \kappa)$ is also determined by a pair of related constraints. From a topological viewpoint, the Boltzmann distribution is equivalent to finding a preferred cycle representative for any homology class, as the latter is a quotient of the former. This linear operator is subject to:

1. the cycle $\rho_{B}([\gamma])$ lies in the same homology class as $[\gamma]$ itself; that is, $\left[\rho_{B}([\gamma])\right]=[\gamma]$, and

2. The inner product $\left(\rho_{B}([\gamma] ; \hat{E}(\tau), \kappa), \partial \alpha\right)_{\hat{E}(\tau), \kappa}=0$ for any $\alpha \in C_{d-1}(X ; \mathbb{R})$.

These operators are defined in subsections 4.2 and 4.3. The two sets of conditions defining $A$ and $\rho_{B}$ are dual. This symmetry allows a single, universal construction to be given for both. In this case, the construction is known as a pseudo-inverse [4]. This is a one-sided inverse (see the first condition in both), subject to an additional constraint (the second condition in both). This allows us to write both operators as a sum indexed over subcomplexes of $K$, giving these physical constructions intrinsic geometric meaning.

There is a tremendous amount of topological and combinatorial information about the Morse complex, and hence the stochastic dynamics of the process, contained in these two operators. They are both written in terms of higher dimensional version of spanning trees and vertices generalized from graphs. Together, they combine to yield

$$
J([\gamma], \kappa, \tau)=A(\tau) \dot{\rho}_{B}([\gamma], \tau),
$$

a computable version of the current density. This allows us to write down the explicit integral representation for the average current density as

$$
Q([\gamma], \kappa, u(\tau))=\int_{0}^{1} d \tau J(\tau) .
$$

In order to obtain meaningful results and have some hope of computing Eq. (8), we must restrict the parameters we allow for the process. Specifically, we narrow our parameters to those which satisfy a generic, nondegeneracy condition. A driving protocol is very nondegenerate if, at any moment in time, either $\hat{E}$ or $\hat{W}$ satisfies one of the following (in place of $f$ )

$$
\sum_{s \in S} f(s) \neq \sum_{t \in T} f(t), \quad \text { for all } S, T
$$


where $S$ and $T$ are subsets of the $(d-1)$-cells (for $\hat{E}$ ) or $d$-cells (for $\hat{W}$ ). This condition is a higher analogue for a function to be injective, or one-to-one, and recovers this notion if $S$ and $T$ are taken to be singletons. This condition, and therefore this subspace of driving protocols, is generic and so there is little lost by this restriction. We shall restrict to very non-degenerate driving protocols for the remainder of this text.

It is easy to see that a non-stationary driving protocol can be arranged to alternate between segments where either $\hat{E}$ or $\hat{W}$ (or both) satisfy Eq. (9); segments for which $\hat{E}$ satisfies this are known as type $E$, and those for $\hat{W}$ are type $W$. The difference between these two segments is visible in the stochastic dynamics. On segments of type of $E$, the evolving cycle will tend to a configuration of minimal 'energy', i.e., it will minimize $\sum_{b} E_{b}$ over the cycle, and be entirely supported on a uniquely determined spanning co-tree. On a type $W$ segment, the cycle can move quite substantially across the complex, but in a way so that its motion is restricted to a specific spanning tree. In fact, it will move along the spanning tree from one spanning cotree to another, with all of these structures being uniquely determined in the low-temperature, adiabatic limit. This motion of moving from co-tree to co-tree along trees is in direct analogy with (and should be thought of as) a particle moving on a graph by jumping from vertex to vertex along a (traditional) spanning tree. On the type $E$ segments, where the cycle is restricted to a single spanning co-tree, there is obviously no current generated. On the type $W$ segments, where the cycle is transitioning from one spanning co-tree to another, current is generated and the values this current takes are determined by the relevant structures involved. Analyzing this motion, we are able to write down an explicit formula for the average current in these limits, and conclude that the coefficients obtained are rational. That is, the main result of this text is the following quantization result:

$$
\lim _{\kappa \rightarrow 0} \lim _{\tau_{D} \rightarrow \infty} Q([\gamma], \kappa, \tau) \in H_{d}(K ; \mathbb{Q}) .
$$

\section{Markov Chain Models for Empirical Currents in the Weak-Noise Limit}

Similar to the case of standard empirical currents, considered in some detail in [11], the problem of higherdimensional empirical current generation for moderate topological driving that satisfies the Morse-Smale condition, in the weak-noise limit can be reduced to Markov Chain (MC) models. This reduction will be addressed with some detail elsewhere. Instead, in this section we introduce the MC models using intuitive physical arguments, with the relevant transition (hopping) rates considered as phenomenological constants, in the case when the driving is topological, which means that the deterministic (advection) component $u$ in the Langevin equation is given by $u^{j}(x)=g^{j k}(x) F_{j}(x)$ with $\partial_{i} F_{j}(x)-\partial_{j} F_{i}(x)=0$.
We can recast this in an equivalent form as $\mathcal{Q}\left(F_{j} \Theta^{j}\right)=0$. Moderate driving means that the critical subspace, which contains of points with $F(x)=0$, is represented by a finite set of isolated non-degenerate (the matrix $\partial_{i} F_{j}(x)$ is not degenerate) critical points. Recall that the number of unstable modes near a critical point, i.e., the number of negative eigenvalues of $\partial_{i} F_{j}(x)$, is referred to as the Morse index of a critical point.

We will also assume the Morse-Smale (MS) condition [19] to be satisfied. Note that the MS condition is usually formulated for gradient flows, however, all results immediately apply to our case of topological driving (i.e., locally gradient flow) with the simple critical structure of moderate driving, as described above. This follows from the fact that within the cells, associated with critical points, the flow is gradient, since the cells are contractible by definition. Let $K_{0}, K_{1}, \ldots, K_{m}$ be the (finite) sets of critical points with a given Morse index. The most important property of a flow satisfying the MS condition is that it provides a cellular (Morse) decomposition of the configuration space $X$, in a way that each critical point $y \in K_{n}$ provides a $n$-dimensional cell $W_{y}$, produced by the unstable manifold of $y$ (i.e., $W_{y}$ consists of all points covered by the flow that starts at $y$ in all unstable directions). We will fix the orientations for all cells in some arbitrary way by choosing the orientations of the unstable subspaces at the corresponding critical points [28],[29],[30]. The cells can be properly compactified (by inspecting the infinite-time flow behavior) so that the border $\partial W_{y}$ is represented by a union of a number of cells related to critical points of index $(n-1)$. If $W_{y^{\prime}} \subset \partial W_{y}$ for some critical point $y^{\prime} \in K_{n-1}$, the intersection $p_{y y^{\prime}}$ of the unstable manifold of $y$ with the stable manifold of $y^{\prime}$ is represented by a flow trajectory that starts at $y$ and terminates at $y^{\prime}$ in infinite time. The set of such trajectories is denoted $P_{y y^{\prime}}=\left\{p_{y y^{\prime}}\right\}$. With each trajectory $p_{y y^{\prime}} \in P_{y y^{\prime}}$ we can associate a binary variable $s\left(p_{y y^{\prime}}\right) \in \mathbb{Z}_{2}$, so that $s\left(p_{y y^{\prime}}\right)=0$ or $s\left(p_{y y^{\prime}}\right)=1$, when the orientation of $W_{y^{\prime}} \subset \partial W_{y}$ matches or mismatches the orientation of $W_{y}$, respectively. The set $C_{\bullet}(K)=\mathbb{Z}\left(K_{\bullet}\right)$ of abelian groups, referred to as the groups of chains is equipped with a set $\partial_{\bullet}: C_{\bullet}(K) \rightarrow C_{\bullet-1}(K)$ of boundary operators, defined by

$$
\partial_{\bullet} y=\sum_{y^{\prime} \in K_{\bullet}-1} \sum_{p \in P_{y y^{\prime}}}(-1)^{s(p)} y^{\prime}, \quad \text { for } y \in K_{\bullet},
$$

so that $\left(C_{\bullet}(K), \partial_{\bullet}\right)$ forms a complex, referred to as the Morse complex, associated with the described above Morse decomposition.

The above the Morse decomposition provides a set of bipartite graphs $G_{n}=\left(K_{n+1}, K_{n} ; E_{n}\right)$ for $n=0,1, \ldots, m-1$ with the edge sets $E_{n}=\bigcup_{\left(y, y^{\prime}\right) \in K_{n+1} \times K_{n}} P_{y y^{\prime}}$. Stated in words, the two types of nodes in bipartite $G_{n}$ are given by critical points of index $(n+1)$ and $n$, whereas the edges represent the flow trajectories that start at $K_{n+1}$-nodes and terminate at $K_{n}$-nodes. The graph $G_{n}$ plays a crucial role in the description of slow stochastic dynamics of 
$n$-cycles and corresponding $n$-dimensional current generation in the weak-noise limit of moderate topological driving that satisfies the MS condition. In the above limit the problem can be reduced to a standard MC process on an infinite oriented graph $\mathcal{G}_{n}=\left(\mathcal{N}_{n}, \mathcal{E}_{n}\right)$. We will first build the graph $\mathcal{G}_{n}$ and define a MC process on it, and after that provide a physical motivation for such a reduction. The reduction (including deriving explicit expressions for the hopping rates) will be addressed elsewhere.

We will denote the nodes of the two types $\alpha \in K_{n+1}$ and $j \in K_{n}$ by Greek and Latin letters, respectively. The nodes of $\mathcal{G}_{n}$ that describe the metastable states of slow stochastic dynamics are given by $\mathcal{N}_{n}=\operatorname{ker} \partial_{n} \subset C_{n}(K)$, which means that a metastable state $\boldsymbol{n} \in \mathcal{N}_{n}$ is an integer vector with the components $n_{j} \in \mathbb{Z}$ and $j \in K_{n}$. The (directed) edges (that belong to $\mathcal{E}_{n}$ ) correspond to elementary hopping events. The edges are partitioned in groups labeled by $\left(j, \alpha ; p_{\alpha j}\right)$ with $j \in K_{n}, \alpha \in K_{n+1}$, and $p_{\alpha j} \in P_{\alpha j}$, i.e., $\mathcal{E}_{n}=\bigsqcup \mathcal{E}_{j \alpha, p_{\alpha j}}^{n}$. All elementary hopping processes that go along the edges of the same group have the same hopping rate denoted by $k_{\alpha j, p_{\alpha j}}$. This means that an elementary process has a beginning $j \in K_{n}$ related to a metastable state, a "transition' state $\alpha \in K_{n+1}$ and a "pathway" $p_{\alpha j} \in P_{\alpha j}$ that connects the beginning to the "transition" (a more adequate physical explanation will be given later in this section). To complete the description of our MC model we should describe all edges that outgo from any given node. Consider an arbitrary node $\boldsymbol{n} \in \mathcal{E}_{n}$ and a group $\mathcal{E}_{j \alpha, p_{\alpha j}}^{n}$ of edges labeled by $\left(j, \alpha ; p_{\alpha j}\right)$. If $n_{j}=0$ there are no edges in the group that outgoes from $\boldsymbol{n}$. If $n_{j} \neq 0$ there is exactly one edge that outgo from $\boldsymbol{n}$. The node $\boldsymbol{n}^{\prime}$, where this unique edge is incoming, can be described as follows:

$$
\begin{aligned}
& n_{k}^{\prime}=n_{k}-\sum_{r \in P_{\alpha k}}(-1)^{s\left(p_{\alpha j}\right)+s(r)} n_{j}, \\
& \boldsymbol{n}^{\prime}=\boldsymbol{n}-(-1)^{s\left(p_{\alpha j}\right)} \partial_{n+1} \boldsymbol{n} .
\end{aligned}
$$

The sign factor $(-1)^{s(p)+s(r)}= \pm 1$ reflects the relative orientation of the cells $W_{j} \subset W_{\alpha}$ and $W_{k} \subset W_{\alpha}$ with respect to $W_{\alpha}$. The second relation in Eq. (12) recasts the first relation using the definition of the boundary operator [Eq. (11)] and clearly demonstrates the fact that the homology class of the state $[\boldsymbol{n}]$ is preserved in the described MC process.

We are now in a position to present the physical motivation that stands behind the described above MC process on the graph $\mathcal{G}_{n}$. The physical picture is based on the time scale separation. The initial (fast) relaxation occurs on a finite time scale in the $\kappa \rightarrow 0$ limit. During the initial relaxation, the initial cycle evolves essentially deterministically and as a result gets aligned with the $n$ dimensional cells $W_{j}$, while performing weak fluctuations in a small neighborhood $U_{n} X \supset K^{(n)}=\bigcup_{j \in K_{n}} W_{y}$ of the the $n$-skeleton $K^{(n)}$ associated with the Morse decomposition of $X$. After the fast relaxation is completed, the evolution of the cycle is represented by a sequence of rare events during which the relevant fragments of the cycle move along the $(n+1)$-cells $W_{\alpha}$. Stated more formally, most of the time the cycle ( $n$-dimensional surface in $X$ ) is restricted to $U_{n} X$, whereas while performing the rare transitions the cycle is restricted to a broader subspace $U_{n+1} X \supset U_{n} X$. Since a transition is rare, it occurs as an optimal fluctuation (that maximizes the probability of the event) according to the following scenario. A fluctuation, represented by a Langevin vector field $\xi$ occurs in the vicinity of a critical point $j \in K_{n}$ that drags a small fragment of the cycle $\zeta: N \rightarrow X$ located near $j \in X$ (the fragment is typically folded) along the path $p_{\alpha j} \in P_{\alpha j}$ to a critical point $\alpha \in K_{n+1}$ that labels a cell $W_{\alpha}$ attached to the cell $W_{y}$. Once the fragment passes the critical point no more noise is required to complete the transition: the fragment, that has been dragged, acquires intersections with the paths $p_{\alpha k} \neq p_{\alpha j}$ and, on the time scale of fast relaxation, the intersections, typically represented by isolated points, will relax to the critical points $k \in W_{n}$ going along the paths $p_{\alpha k}$, mentioned above. As a result the whole bigger fragment of the cycle that used to be located in a small neighborhood of $W_{j}$ will locate itself in the small neighborhoods of the cells $W_{k}$, for all $k \in K_{n}$ such that $W_{k} \subset \partial W_{\alpha}$. Note that during the whole transition the whole fragment moves along a small neighborhood of the "transition" $(n+1)$-cell $W_{\alpha}$.

The reduced variables $\boldsymbol{n} \in \mathcal{N}_{n}$ that characterize the metastable states and form the nodes of the graph $\mathcal{G}_{n}$ introduced above are of topological nature. They can be described as follows. Consider a metastable configuration of our cycle $\zeta$, when it is located in the small neighborhood $U_{n} X \supset K^{(n)}$ of the $n$-skeleton of $X$. We further take a critical point $j \in K_{n}$, look at all components of our cycle located near $W_{j}$ (typically the cycle is folded) and define $n_{j}$ as the number of times the cycle appears in the neighborhood with the same orientation as $W_{j}$ minus the number of times it appears with the opposite orientation. This can be formulated more precisely by defining $n_{j}$ as the intersection index of $\zeta: N \rightarrow X$ with the stable manifold of the critical point $j$. The transverse size of $U_{n} X$, where a metastable cycle is concentrated, is $\sim \sqrt{\kappa}$. Therefore, if the correlation length of the random field $\xi$ is large compared to $\sqrt{\kappa}$ (which will always happen for low enough noise), all components of the cycle will be dragged through the transition state together. On the other hand, if the correlation length is small compared to the cell sizes, the elementary transitions will be restricted to individual cells $W_{\alpha}$. This rationalizes the description in terms of the reduced topological variables $\boldsymbol{n}$, as well as the structure of the elementary transitions, described by Eq. (12).

The fact that the obtained MC process occurs on an infinite graph reflects the field-theory nature of the original problem. We have demonstrated, however, that the moments of the current distributions, including the stationary current can be calculated using spectral decompositions for operators acting in the spaces of states (referred to as the spaces of correlation functions) associated with 
finite-dimensional manifolds. Naturally such a reduction is inherited by the MC models, i.e., the moments of the current distributions can be calculated by using spectral decompositions in finite-dimensional vector spaces that describe the corresponding correlation functions.

Let $\mu(\boldsymbol{n})$ be the probability distribution that describes the $\mathrm{MC}$ process on $\mathcal{G}_{n}$, where it satisfies the appropriate master equation. We start with the stationary current that can be expressed in terms of a correlation function (reduced distribution) that belongs to $\mathbb{R}\left(K_{n}\right)$ and is described by a vector with the components

$$
\rho_{i}=\sum_{\boldsymbol{n}} n_{i} \mu(\boldsymbol{n})
$$

By calculating the time derivative $\dot{\rho}_{j}$ via applying the full master equation for $\mu(\boldsymbol{n})$ we obtain, after some straightforward transformations, a closed equation for $\rho$ that has a natural form

$$
\begin{aligned}
\dot{\rho}_{i} & =\sum_{\alpha \in K_{n+1}} \sum_{j \in K_{n}}^{j \neq i} \sum_{p \in P_{\alpha j}} \sum_{r \in P_{\alpha i}} k_{\alpha j, p}(-1)^{s(p)+s(r)} \rho_{j} \\
& -\sum_{\alpha \in K_{n+1}} \sum_{p \in P_{\alpha i}} k_{\alpha i, p} \rho_{i} .
\end{aligned}
$$

The only trick used in deriving Eq. (14) is introducing the axillary correlation functions

$$
\rho_{i}^{(j)}=\sum_{\boldsymbol{n}}^{n_{j} \neq 0} n_{i} \mu(\boldsymbol{n})
$$

and appreciating $\rho_{i}^{(i)}=\rho_{i}$.

\section{Generated Higher-Dimensional Currents in a Periodically Driven System on a CW complex}

In this section, we develop the necessary tools and give an explicit integral representation for the average current density generated per period of driving protocol. Our method relies on the reduction to the Morse CW complex $K$ of $X$ discussed in the previous section. This is a generalization of the one-dimensional case, given by writing the SFP operator in a geometric fashion on the chain complex of $K$. We then write the integral representation of the generated current in terms of two key components, the solution to Kirchhoff's network problem and a higherdimensional version of the Boltzmann distribution, both of which are familiar from the classical, one-dimensional case. In subsection 4.2, we discuss Kirchhoff's network problem and its solution, given by application of the aforementioned theory of pseudo-inverses. The second component is the Boltzmann distribution, studied in subsection 4.3. After obtaining both expressions in higher dimensions, we combine them to yield the desired result in subsection 4.4.

\subsection{Integral Representation for the Average Generated Current}

The first goal is to express Eq. (14) in terms of a supersymmetric Fokker-Planck operator on the CW complex $K$. We begin by constructing an adjoint to the boundary map $\partial: C_{n}(K) \rightarrow C_{n-1}(K)$ defined in Eq. (11). The boundary map should be thought of as a discrete analogue of the divergence operator from calculus, and so the adjoint map $\partial^{\dagger}$ should be thought of as a gradient operator. It is given by

$$
\partial^{\dagger} y^{\prime}=\sum_{y \in S_{n}} \sum_{p \in P_{y y^{\prime}}}(-1)^{s(p)} y \quad y \in S_{n-1} .
$$

Both $\partial$ and $\partial^{\dagger}$ are useful operators for our formulation since their definitions are orientation-independent. Furthermore, they provide concise definitions of the average current density.

All that remains for the SFP operator are the rates of the process. We are focused with the case when the transition rates satisfy detailed balance at each moment in time. We write them in Arrhenius form

$$
k_{i \alpha, p}(t)=e^{-\kappa^{-1}\left(W_{\alpha}(t)-E_{i}(t)\right)}
$$

where $k_{i \alpha, p}$ is the rate of hopping along the pathway $p$, starting at cell $i$, and transitioning over $\alpha$. We encorporate these rates as linear operators by defining diagonal matrices $\hat{E}(t)=\operatorname{diag}\left(E_{1}(t), \ldots, E_{\left|K_{d-1}\right|}(t)\right)$ and $\hat{W}(t)=\operatorname{diag}\left(W_{1}(t), \ldots, W_{\left|K_{d}\right|}(t)\right)$. These definitions combine to allow us to define the SFP operator on $C \bullet(K)$ as

$$
\hat{H}(\gamma(t)):=\partial e^{-\kappa^{-1} \hat{W}(t)} \partial^{\dagger} e^{\kappa^{-1} \hat{E}(t)} .
$$

It is a straightforward application of the above definitions to verify that $\hat{H} \rho$ coincides with the r.h.s. of Eq. (14). Therefore, we succinctly recast Eq. (14) as

$$
\hat{H}(t) \rho(t)=\dot{\rho}(t),
$$

resembling the Fokker-Planck equation from Langevin dynamics. Furthermore, this serves as a discrete analog to the SFP equation considered in [6].

We are primarily interested in the average current density over many periods of the driving protocol. The average current density $Q$ is given by

$$
Q(\gamma(t))=\frac{1}{N} \int_{0}^{N \tau_{D}} d t J(t), \quad N \gg 0 .
$$

There is an obvious adiabatic theorem which holds true in this generalized setting. It has been treated mathematically in [8], and states that a periodic solution to the SFP equation exists and is unique. Furthermore, in the adiabatic limit, this solution tends to the Boltzmann distribution. The remainder of this section is devoted to showing Eq. (7) is valid. An explicit formula for both $A$ and $\rho^{B}$ will be given in the next two subsections. Combining these two pieces, we obtain the desired formula

$$
Q(\gamma(t))=\int_{0}^{1} d \tau A(\tau) \dot{\rho}^{B}(\tau), \quad \tau_{D} \rightarrow \infty,
$$


where we have expressed the quantities in terms of dimensionless time $\tau=t / \tau_{D}$.

\subsection{Higher-Dimensional Network Kirchhoff Theorem}

The classical Kirchhoff problem aims to find the unique current in a resistive network given some initial current. The higher dimensional network problem asks the analogous question for CW complexes of arbitrary dimension.

The higher dimensional Kirchhoff problem, i.e. constructing a solution $A=A_{\hat{W}(t), \kappa}$ to Eq. (5), is solved by introducing the discrete counterpart to the continuous pseudo-inverse introduced in [6], which acts on $(d-1)$ boundaries to produce $d$-chains. We will write down the operator $A$ explicitly in terms of higher dimensional spanning trees, introduced in subsection 2.1 (we omit the dependence of $A$ on $\hat{W}(t)$ and $\kappa$ in what follows). Fix a spanning tree $T$, and define an operator $A_{T}$ as follows. By definition, every boundary in $X$ is the boundary of something in $T$. Or in symbols, for every $b=\partial \alpha \in C_{d-1}(X ; \mathbb{R})$, there is a unique $d$-chain in $T, A_{T}^{b}$, so that $\partial A_{T}^{b}=b$. Define $A_{T}(b)=A_{T}^{b}$. The solution to Kirchhoff's higher dimensional network theorem is then given by taking a weighted sum of such operators, indexed over the set of all spanning trees. The final form the operator takes is

$$
A=\frac{1}{\Delta} \sum_{T} w_{T} A_{T}
$$

where $w_{T}$ is the weight of a tree, as defined in subsection 2.2 , and $\Delta=\sum_{T} w_{T}$. It is straightforward to verify that this definition of $A$ verifies the criteria of Eq. (5). The first property follows directly from the definition of $A_{T}$, and the second follows by noting that $A$ is self-adjoint with respect to the inner product of Eq. (6).

The operator $A_{T}$ has been given in a geometric fashion in terms of unique chains in the spanning tree $T$. This is a direct generalization of the Kirchhoff theorem for graphs, or one-dimensional complexes. In this case, the operator $A_{T}$ sends a boundary, say given by a difference of vertices $j-i$, to the unique path in the (ordinary) spanning tree $T$ from $i$ to $j$. In fact, the unique current in a resistive network, i.e. a solution to Kirchhoff's problem on graphs, is given by Eq. (22).

\subsection{Higher-Dimensional Boltzmann Distributions and Kirchhoff Co-Tree Theorem}

The other key ingredient needed for Eq. (21) is the higher dimensional Boltzmann distribution. This is a direct generalization of the classical distribution from statistical mechanics.

The higher dimensional Boltzmann problem (also known in topology as the Hodge decomposition problem) is to find an explicit cycle representative for any homology class. The Boltzmann distribution can be written explicitly in terms of spanning co-trees, introduced in subsection 2.2. By their definition, within each co-tree we can find a unique cycle representative for each homology class of $X$.
Therefore, for each spanning co-tree $L$, define $\psi_{L}([\gamma])$ to be the unique cycle representative of $[\gamma]$ in $L$. We then take a weighted sum over these operators to obtain

$$
\rho_{B}=\frac{1}{\Lambda} \sum_{L} \tau_{L} \psi_{L}
$$

where $\tau_{L}$ is the weight of the spanning co-tree $L$ as in subsection 2.2 and $\Lambda=\sum_{L} \tau_{L}$. As previously mentioned, this problem (and its solution) are dual to the Kirchhoff problem, so the same proof used there applies here. A rigorous proof of this fact without duality was given in [7] using pseudo-inverses.

An important case to keep in mind is the familiar one of a graph, or one-dimensional $\mathrm{CW}$ complex. In this case, the spanning co-trees are given by the vertices, $c_{L}=1$, and each vertex $x$ is then weighted with $\tau_{x}=e^{-\beta E_{x}}$. In this case, Eq. (23) becomes

$$
\rho_{B}=\frac{\sum_{x \in X_{0}} e^{-\beta E_{x}} \cdot x}{\sum_{x \in X_{0}} e^{-\beta E_{x}}},
$$

reproducing the famous distribution.

\subsection{Low-Temperature Adiabatic Limit: Explicit Expres- sion for the Generated Current}

We are now in a position to verify Eq. (21). The equivalence between the expressions given in Eq. (4) and Eq. (7) can be seen by a straightforward argument. Consider the set of all $d$-chains $\nu \in C_{d}(X ; \mathbb{R})$ such that

$$
\partial \nu=\dot{\rho}, \quad(\nu, z)_{\hat{W}(\tau), \kappa}=0,
$$

for every $z \in Z_{d}(K)$. First, it is clear that any $\nu$ satisfying these two conditions must be unique. If $\nu$ and $\nu^{\prime}$ were both solutions, then the first condition of Eq. (25) implies $\partial\left(\nu-\nu^{\prime}\right)=0$, so that $\nu-\nu^{\prime}$ would be a cycle. The second condition then implies $\nu-\nu^{\prime}$ to be orthogonal to itself, forcing $\nu=\nu^{\prime}$. It remains to show that the each of the aforementioned expressions satisfy these two criteria, after which the proof shall be complete. For Eq. (4), the two conditions are verified in precisely the same manner as in the continuous case (Eqs. (56) and (57) in [6]). For Eq. (7), both conditions are readily verified by the definition of $A$ in Eq. (5).

\section{Low-Temperature Adiabatic Limit: Quantiza- tion of Generated Currents}

We now state and prove the main result on the current generated under a multi-dimensional Langevin process: rational quantization. The quantization relies on restricting to the generic set of very non-degenerate driving protocols, defined in subsection 2.2. This restriction allows for a clear analysis in terms of two distinct types of segments of the driving protocol. We take the adiabatic limit for the remainder of this section, so that $N \rightarrow \infty$. 
On a segment of type $W$, only the $W$ parameters are changing, and the functional $E$ is constant. With these parameters, the evolving fragment will remain fixed and no current will be generated. This can be seen explicitly in Eq. (21), by noting that if $E$ is fixed, then $\dot{\rho}_{B} \rightarrow 0$ and $\kappa \rightarrow 0$ :

$$
\lim _{\kappa \rightarrow 0} Q=\int_{a}^{b} d \tau A \dot{\rho}_{B}=0
$$

On a type $E$ segment, the functional $E$ is varying and $W$ is fixed. It is with these parameters that average current density can be generated. Even more, on an individual type $E$ segment, the average current density is determined by the driving protocol at the end points. In the limit as $\kappa \rightarrow 0$, the linear map $A \rightarrow A_{T}$, where $T$ is determined uniquely to be the tree which minimizes

$$
\sum_{\alpha \in T} W(\alpha)
$$

Therefore, in the low-noise limit, we have

$$
\begin{aligned}
\lim _{\kappa \rightarrow 0} Q & =\lim _{\kappa \rightarrow 0} \int_{c}^{d} d \tau A \dot{\rho}_{B} \\
& =A_{T} \int_{c}^{d} d \tau \dot{\rho}_{B} \\
& =A_{T}\left(\psi_{L(d)}-\psi_{L(c)}\right),
\end{aligned}
$$

where $L(c)$ and $L(d)$ are the unique spanning co-tree at their corresponding values of driving protocol. As previously mentioned, each of these individual pieces are weighted with only rational numbers. Summing over all alternations of segments, the average current density will only take on rational numbers or the value 0 . Therefore, the average current density will be a rational number in the low-temperature, adiabatic limit, completing the proof.

Although the process we have been describing throughout this text is inherently stochastic, the low-temperature, adiabatic limit allows us to provide an intuitive, albeit overly simplified, picture from the viewpoint of average current density. Suppose the parameters are varying by starting at a segment of type $E$, then type $W$, then back to $E$. On each type $E$ segment, there is a preferred spanning co-tree; call them $L$ and $L^{\prime}$, respectively. On the type $W$ segment, there is a unique spanning tree $T$. By the defining properties of spanning trees, they contain all spanning co-trees; in particular, $T$ contains both $L$ and $L^{\prime}$. On the initial type $E$ segment, the evolving cycle tends to $L$, where it will remain with an overwhelming probability. As the driving protocol transitions to the type $W$ segment, the cycle will begin to fluctuate in the spanning tree $T$. It will continue evolving in $T$, with its motion restricted to the subcomplex $A_{T}^{L-L^{\prime}}$, until it gets to $L^{\prime}$, where it will remain again with overwhelming probability until the next transition.

\section{Discussion and Path Forward}

We have extended the concept of currents and fluxes, generated in non-equilibrium (driven) stochastic processes to higher dimensions. This has been done for both the continuous [6] and discrete cases. In the continuous case the higher-dimensional current characterizes the same process as the standard stochastic currents, i.e., Langevin stochastic dynamics on a manifold $X$ (of arbitrary dimension $\operatorname{dim}(X)=m$ ) with inhomogeneous noise.

The results on discrete stochastic models, associated with CW complexes that are presented in this manuscript, focus on the periodic driving case with emphasis on the adiabatic driving limit, and special emphasis on the effect of quantization of stochastic currents in the lowtemperature adiabatic limit, the effect that in the case of graphs has been observed experimentally [22, 34, 2, 27] and established theoretically $[12,13]$ in our earlier work.

We cannot overly emphasize that, as opposed to the continuous case [6], the discrete setting for stochastic fluxes brings in a new class of discrete stochastic models, which are associated with CW complexes, rather than just graphs. The models associated with CW complexes apparently look like a very artificial and unnatural generalization of Markov chain processes on graphs. On the other hand, the higher-dimensional fluxes, despite needing a variety of mathematical techniques (that are not very traditional in the chemical physics community) to be properly handled, are conceptually very simple. One deals with the same class of Langevin processes, with the only difference that the new observables are associated with stochastic trajectories of extended objects, namely higher-dimensional cycles. Alternatively, discrete models deal with finite-dimensional vector spaces of states and thus are advantageous computationally. This is one of the reasons why we spent so much effort and a variety of techniques on considering the continuous models (sections (2) and (3) in the prequel [6]) so that in section 3 we were able to derive the CW complex models, interpreting them as a computational tool for treating higher-dimensional currents/fluxes in continuous spaces.

The main result presented in this paper, namely, rational quantization of higher dimensional currents in the low-temperature adiabatic limit, has been achieved by applying the following key steps.

(i) We started with deriving an expression for the average flux in the adiabatic limit that generalizes the expression, obtained in our earlier work for Markov periodic processes on graphs, and can be also interpreted as the discrete counterpart of Eq. (36) of [6], the latter describing the flux in the continuous case. The derivation is straightforward and follows the lines of the similar derivation presented in the first manuscript.

(ii) In the graph case the Boltzmann distribution is known explicitly, whereas the pseudo-inverse that solves the Kirchhoff problem is expressed as a weighted sum over the spanning trees of the graph. In the CW complex case 
it was not obvious what an adequate definition of a spanning tree was, since various definitions have been presented in the literature. Besides, identifying an explicit formula for higher-dimensional Boltzmann distributions appeared to be a much less tractable problem. We have made a surprising observation that the Kirchhoff problem and the problem of identification of the Boltzmann distribution are in fact dual to each other, and can be both treated as finding a proper pseudo-inverse. We found a theorem in linear algebra, which seems not to be a part of common knowledge, applied it to both problems, which allowed to identify the adequate notions for spanning trees and co-trees, the latter being objects naturally appearing in representing the pseudo-inverse for the Boltzmann problem.

(iii) We further expressed the coefficients in front of the spanning trees and co-trees in the aforementioned weighted sums over the trees and co-trees, respectively, in terms of topological invariants of the CW complex and its trees and co-trees, which resulted in a formula, expressed in completely topological terms.

(iv) Finally we applied the low-temperature limit, resulting in a purely topological formula that generalizes the expression for Markov chain processes on graphs to the CW complex case. It needs to be stressed that as opposed to integer quantization in the graph case, in a generic situation quantization is rational for CW complex models. Formally it occurs due to additional factors in denominators of the coefficients in front of the spanning trees and co-trees in the higher-dimensional Kirchhoff tree and co-tree theorems, that are due to the torsion (finite cyclic) subgroups of the relevant homology groups of the CW complex, and its relevant spanning tress and co-trees.

The additional complexity of rational quantization can be addressed starting from the discrete CW-models. The nontrivial nature of the higher-dimensional case shows up in discrete model in a less nasty way. We still have a closed equation for the probability distribution, which can be efficiently twisted, however the closed equation involves the infinite-dimensional Hilbert space of states, associated with the infinite graph, introduced in section 3 . On the other hand, in many cases dynamics that involves Hilbert spaces can be efficiently studied, using e.g., spectral methods. In either case, we see that that dimension of the stochastic trajectory is responsible for the additional complexity and not the underlying configuration space.

\section{Acknowledgements}

This material is based upon work supported by the National Science Foundation under Grant No. CHE-1111350.

[1] Shahaf Asban and Saar Rahav. No-pumping theorem for many particle stochastic pumps. Physical Review Letters, 112(5), February 2014.

[2] R. Dean Astumian. Adiabatic operation of a molecular machine. Proceedings of the National Academy of Sciences, 104(50):19715-19718, December 2007.
[3] R Dean Astumian. Design principles for brownian molecular machines: how to swim in molasses and walk in a hurricane. Physical Chemistry Chemical Physics, 9(37):5067-5083, 2007.

[4] Adi Ben-Israel and Thomas N. E. Greville. Generalized inverses. CMS Books in Mathematics/Ouvrages de Mathématiques de la SMC, 15. Springer-Verlag, New York, second edition, 2003.

[5] J-P Bouchaud and Rama Cont. A langevin approach to stock market fluctuations and crashes. The European Physical Journal B-Condensed Matter and Complex Systems, 6(4):543-550, 1998.

[6] Michael J Catanzaro, Vladimir Y Chernyak, and John R Klein. Stochastic dynamics of extended objects in driven systems: I. higher-dimensional currents in the continuous setting. Submitted to Chem. Phys.

[7] Michael J Catanzaro, Vladimir Y Chernyak, and John R Klein. A higher boltzmann distribution. arXiv preprint arXiv:1506.06775, 2015.

[8] Michael J. Catanzaro, Vladimir Y. Chernyak, and John R. Klein. Dynamics and fluctuations of cellular cycles on cw complexes. In preparation, 2016.

[9] Vladimir Chernyak, Michael Chertkov, Ildar Gabitov, Igor Kolokolov, and Vladimir Lebedev. Pmd-induced fluctuations of bit-error rate in optical fiber systems. Journal of lightwave technology, 22(4):1155, 2004.

[10] Vladimir Y Chernyak, Michael Chertkov, Joris Bierkens, and Hilbert J Kappen. Stochastic optimal control as nonequilibrium statistical mechanics: calculus of variations over density and current. Journal of Physics A: Mathematical and Theoretical, 47(2):022001, 2013.

[11] Vladimir Y Chernyak, Michael Chertkov, Sergey V Malinin, and Razvan Teodorescu. Non-equilibrium thermodynamics and topology of currents. Journal of Statistical Physics, 137(1):109$147,2009$.

[12] Vladimir Y Chernyak, John R Klein, and Nikolai A Sinitsyn. Quantization and fractional quantization of currents in periodically driven stochastic systems. i. average currents. The Journal of chemical physics, 136(15):154107, 2012.

[13] Vladimir Y Chernyak, John R Klein, and Nikolai A Sinitsyn. Algebraic topology and the quantization of fluctuating currents. Advances in Mathematics, 244:791-822, 2013.

[14] Michael Chertkov, Michael Fisher, Scott Backhaus, Russell Bent, and Sidhant Misra. Pressure fluctuations in natural gas networks caused by gas-electric coupling. In System Sciences (HICSS), 2015 48th Hawaii International Conference on, pages 2738-2747. IEEE, 2015.

[15] William T Coffey, Yu P Kalmykov, and JT Waldron. The langevin equation with applications to stochastic problems in physics, chemistry and electrical engineering second edition. World scientific series in contemporary chemical physics, 14, 1996.

[16] Sybren Ruurds De Groot and Peter Mazur. Non-equilibrium thermodynamics. Courier Corporation, 2013.

[17] Daniel T Gillespie. The chemical langevin equation. The Journal of Chemical Physics, 113(1):297-306, 2000.

[18] Takahiro Hatano and Shin-ichi Sasa. Steady-state thermodynamics of langevin systems. Physical review letters, 86(16):3463, 2001.

[19] Morris W Hirsch. Differential topology, volume 33. Springer Science \& Business Media, 2012.

[20] C Jarzynski. Equilibrium free energies from nonequilibrium processes. Acta Physica Polonica. Series B, 29(6):1609-1622, 1998.

[21] Min G. Lee, Akihiko Oba, and Hideki Takayasu. Parameter Estimation of a Generalized Langevin Equation of Market Price, pages 260-270. Springer Japan, Tokyo, 2002.

[22] David A Leigh, Jenny KY Wong, François Dehez, and Francesco Zerbetto. Unidirectional rotation in a mechanically interlocked molecular rotor. Nature, 424(6945):174-179, 2003.

[23] Don S Lemons and Paul Langevin. An introduction to stochastic processes in physics. JHU Press, 2002.

[24] Sergey V. Malinin and Vladimir Y. Chernyak. Transition times 
in the low-noise limit of stochastic dynamics. The Journal of Chemical Physics, 132(1):014504, January 2010.

[25] A. Nerode and H. Shank. An Algebraic Proof of Kirchhoff's Network Theorem. The American Mathematical Monthly, 68(3):244-247, March 1961

[26] Bernt Oksendal. Stochastic differential equations: an introduction with applications. Springer Science \& Business Media, 2013.

[27] Juan MR Parrondo. Reversible ratchets as Brownian particles in an adiabatically changing periodic potential. Physical Review E, 57(6):7297, 1998.

[28] Lizhen Qin. On moduli spaces and cw structures arising from morse theory on hilbert manifolds. J. Topol. Anal., pages 459526,2010 .

[29] Lizhen Qin. An application of topological equivalence to morse theory. arXiv preprint, arXiv:1102.2838, 2011.

30] Lizhen Qin. On the associativity of gluing. arXiv preprint arXiv:1107.5527, 2011.

31] Saar Rahav, Jordan Horowitz, and Christopher Jarzynski. Directed flow in non-adiabatic stochastic pumps. Physical Review Letters, 101(14), October 2008. arXiv: 0808.0015.

[32] Saar Rahav, Jordan Horowitz, and Christopher Jarzynski. Directed flow in nonadiabatic stochastic pumps. Physical review letters, 101(14):140602, 2008

[33] Hannes Risken. Fokker-planck equation. In The Fokker-Planck Equation, pages 63-95. Springer, 1984.

[34] N. A. Sinitsyn. Stochastic pump effect and geometric phases in dissipative and stochastic systems. Journal of Physics A: Mathematical and Theoretical, 42(19):193001, May 2009. arXiv: 0903.4231.

[35] NA Sinitsyn and Ilya Nemenman. The berry phase and the pump flux in stochastic chemical kinetics. EPL (Europhysics Letters), 77(5):58001, 2007.

[36] Nicolaas Godfried Van Kampen. Stochastic processes in physics and chemistry, volume 1. Elsevier, 1992

[37] Hermann Weyl. Repartici on de corriente en una red conductora. Revista Matematica Hispano-Americana, (5):153-164, 1923. 